\title{
The role of CDK1 in apoptin-induced apoptosis in hepatocellular carcinoma cells
}

\author{
JING ZHAO ${ }^{1}$, SU-XIA HAN ${ }^{1}$, JIN-LU MA ${ }^{1}$, XIA YING ${ }^{1}$, PEIJUN LIU ${ }^{2}$, JUAN LI $^{2}$, \\ LIJUAN WANG $^{1}$, YING ZHANG ${ }^{1}$, JIGUANG MA ${ }^{1}$, LI ZHANG ${ }^{1}$ and QING ZHU ${ }^{1}$ \\ ${ }^{1}$ Department of Oncology, ${ }^{2}$ Center for Translational Medicine, The First Affiliated Hospital, \\ Xi'an Jiaotong University Medical College, Xi'an, Shaanxi 710061, P.R. China
}

Received January 30, 2013; Accepted March 5, 2013

DOI: $10.3892 / o r .2013 .2426$

\begin{abstract}
Apoptin, a small protein derived from the chicken anemia virus, specifically induces apoptosis in transformed cells or tumor cells but not in normal cells. Thus, apoptin is involved in a general, tumor-specific pathway. Apoptininduced apoptosis presumably requires additional interaction partners that activate specific signaling pathways in cancer cells. A number of molecules interact with apoptin and play an important role in the nuclear localization of apoptin or its tumor-selective cytotoxicity. Our data indicated that apoptin selectively kills HepG2 hepatocellular carcinoma (HCC) cells but has no effect on the normal liver cell line HL-7702. Analyses of human HCC tissue samples confirmed that CDK1 (cyclin-dependent kinase 1) activity was detected in primary malignancies but not in healthy paraneoplastic tissues. shRNA knockdown of CDK1 significantly reduced the tumor-specific killing effects of apoptin, suggesting that CDK1 plays an important role in the regulation of apoptin-induced apoptosis. Furthermore, the majority of apoptin translocated to the cytoplasm from the nucleus after knockdown of CDK1. Collectively, our results revealed for the first time that apoptin interacts with CDK1 in the complex process of tumorigenesis. The link between CDK1 and apoptin may be a novel cellular signaling pathway to modulate apoptosis in cancer; therefore, apoptin may have pharmacological potential to be directly employed for cancer therapy.
\end{abstract}

\section{Introduction}

Hepatocellular carcinoma (HCC) is one of the most common types of cancer in the world. HCC is often diagnosed at an advanced stage as it progresses rapidly due to vascular invasion

Correspondence to: Professor Qing Zhu or Dr Suxia Han, Department of Oncology, The First Affiliated Hospital, Xi'an Jiaotong University Medical College, 277 Yanta West Road, Xi'an, Shaanxi 710061, P.R. China

E-mail: newzhuqing1972@yahoo.com

E-mail: hansuxia22099@yahoo.com.cn

Key words: apoptin, CDK1, hepatocellular carcinoma, apoptosis and multiple metastases (1). HCC patients typically relapse after treatment, which leads to a low 5-year survival rate of less than 7\% (2). Current treatment options for advanced HCC include chemotherapy, radiotherapy, proton beam therapy, chemo-embolization and ablation. However, these treatments remain unsatisfactory. Gene therapy is a relatively new technique in the therapeutic battle against cancer (3).

Apoptin, a small protein that is derived from the chicken anemia virus and consists of 121 amino acids $(4,5)$, has attracted significant attention as it possesses tumor-specific cytotoxicity $(6,7)$. The ectopic expression of apoptin induces apoptosis in various human transformed cells and tumor cells but has little or no cytotoxic effects in several normal human cell lines $(8,9)$. The selective toxicity of apoptin is mainly attributed to its differential subcellular localization in tumor and normal cells $(10,11)$. In cancer cells, apoptin predominantly localizes to the nucleus, whereas in normal cells, its nuclear accumulation is severely impaired. The present study suggested that apoptin requires additional interacting molecular partners that involve specific signaling pathways in cancer cells. Apoptin interacts with various partners of the human proteome including PI3K/AKT $(12,13)$, PKC $\beta$ (14), APC1 (15), and PML (16), which regulate either apoptin activation or execution processes. These recent advances demonstrate that elucidating the mechanism of apoptin-induced apoptosis can lead to novel tumor-specific pathways that may be exploitable as anticancer drug targets.

Using kinase software, we found that CDK1 is a candidate interacting molecule. The HCC cell line HepG2 and the human normal liver cell line HL-7702 were used to study the role of CDK1 in apoptin-induced apoptosis in liver cancer cells. These cell lines showed a marked difference in the expression level of CDK1; CDK1 expression levels in HepG2 cells were significantly higher than in HL-7702 cells. Expression vectors bearing genes encoding GFP-apoptin or Flag-apoptin proteins were developed to evaluate the tumor-specific toxicity of apoptin in HCC. Apoptin selectively killed HepG2 cells but had no effect on HL-7702 cell proliferation. Following shRNA knockdown of CDK1, the tumor-specific killing effect of apoptin was significantly reduced, and the majority of apoptin translocated to the cytoplasm from the nucleus. The results indicated that downregulation of CDK1 leads to the cytoplasmic relocalization of apoptin. 
CDK1 is a highly conserved protein that functions as a serine/threonine kinase and is a key player in cell cycle regulation. CDK1 activity during apoptosis was first identified in lymphoma cells (17). However, the molecular mechanisms of CDK 1 in the processes of apoptosis remain unknown. Thus, deregulation of cell cycle regulators, such as CDK1, likely contributes to the tumorigenesis of HCC.

In the present study, we provided strong evidence for the interaction between CDK1 and apoptin in the process of tumorigenesis. CDK1 plays a role in the accumulation of apoptin in the nucleus. This strategy enabled us to identify apoptin-interacting cellular molecular targets, and the selective accumulation of apoptin in the nucleus of cancer cells may be utilized for oncotherapy development.

\section{Materials and methods}

Patients and specimens. A total of 48 human primary HCC tissues and matched control tissues were obtained from patients who underwent hepatectomy at the the First Affiliated Hospital of Xi'an Jiaotong University Medical college, between 2009 and 2011. The mean age was 50 years, with 18 females and 30 males. None of these patients had received preoperative chemotherapy or radiotherapy. Overall survival, which was defined as the time from the surgery to patient death or the last follow-up, was used as a measure of prognosis. Both the tumor and the corresponding non-tumor tissues not less than $3 \mathrm{~cm}$ away from the HCC tissue were sampled, and the diagnosis was confirmed by pathological examination. After surgical resection, the fresh cancer tissues and matched normal tissues from the HCC patients were obtained for western blot analysis. Histological types were assigned according to the WHO classification criteria. The protocols used in this study were approved by the hospital's Protection of Human Subjects Committee. The use of human tissues in this study was approved by the Institutional Review Board of the First Affiliated Hospital of Xi'an Jiaotong University Medical College and was carried out in accordance with international guidelines, and written informed consent was obtained from each patient.

Cell culture. HepG2, Hep3B, SMMC-7721 and HL-7702 cells were purchased from the Shanghai Institute of Cell Biology (Shanghai, China). The cells were cultured in Dulbecco's modified Eagle's medium (DMEM) supplemented with $10 \%$ fetal bovine serum (FBS), $100 \mathrm{U} / \mathrm{ml}$ penicillin, and $100 \mu \mathrm{g} / \mathrm{ml}$ streptomycin (Gibco, Grand Island, NY, USA) in a humidified incubator at $37^{\circ} \mathrm{C}$ with $5 \% \mathrm{CO}_{2}$.

Expression plasmid construction and transfection. To construct pcDNA3.1-Flag-apoptin and pEGFP-apoptin, fulllength apoptin (including the full 121 codons) was excised from plasmid pcDNA3.1-apoptin-V5 after PCR with EcoRI and BamHI and then cloned into the pcDNA3.1-Flag and pEGFP vectors at the EcoRI and BamHI sites. The 4 complementary primers used were 5'-GCGGATCCGCCACCATGG ATTACAAGGATGACGACGATAAGAACGCTCTCCAAG AAGATA-3' and 5'-GCGAATTCTTACAGTCTTATACA CCTTCTTGC-3', 5'-CGGAATTCGCCACCATGAACGC TCTCCAAGAAGATAC-3' and 5'-CGGGATCCTTACAGT
CTTATACACCTTCTTGC-3'. The fidelity of the plasmid constructs was confirmed by DNA sequencing.

Cells were plated $24 \mathrm{~h}$ before transfection in a 6-well plate at a density of $2 \times 10^{5}$. Transfection with $4.0 \mu \mathrm{g}$ pcDNA3.1-Flagapoptin/pEGFP-apoptin vector or $4.0 \mu \mathrm{g}$ pcDNA3.1/pEGFP empty vector (as a positive control) was performed using Lipofectamine 2000 (Invitrogen, Paisley, UK) according to the manufacturer's protocol.

MTT proliferation. Cell viability was determined by the colorimetric 3-(4,5-dimethylthiazol-2-yl)-2,5-diphenyltetrazolium bromide (MTT) assay. HepG2 and/or HL-7702 cells were transfected with pcDNA3.1-Flag-apoptin/pEGFP-apoptin vector, pcDNA3.1/pEGFP empty vector or siRNA-CDK1. Cells were seeded at $5 \times 10^{4}, 10^{4}$, and $10^{3}$ per well in 96-well plates for MTT assays. On days $0,1,2,3,4$ and 5 post-infection, the cells were washed twice with PBS and incubated with $0.5 \mathrm{mg} / \mathrm{ml}$ MTT (Sigma) for $4 \mathrm{~h}$. After the incubation period, cells were washed with PBS, solubilized with dimethyl sulfoxide (DMSO), and quantified using a microplate reader at the absorbance of $550 \mathrm{~nm}$. The average values were obtained from triplicates.

Flow cytometric analysis. The apoptosis was evaluated by Annexin-V binding and 7-AAD uptake using an Annexin V-PE/7-AAD kit (Keygen, China). Briefly, cells were plated at a density of $1 \times 10^{5}$ cells/well into 6 -well plates for $24 \mathrm{~h}$. The cells were transfected with pEGFP-apoptin vector and shRNA-CDK1 for 24 and $48 \mathrm{~h}$, respectively. The cells were washed three times with cold PBS and resuspended in Annexin $\mathrm{V}$ binding buffer. The cells were stained with Annexin V-PE for $15 \mathrm{~min}$, washed, and then stained with 7-AAD. The cells were analyzed on a BD FACSCanto II (Becton-Dickinson, Oxford, UK) and 10,000 events were acquired per sample. Subsequently, the cells were analyzed by flow cytometry using CellQuest software (BD Biosciences).

Western blot analysis. Whole-cell extracts were prepared from pcDNA3.1-Flag-apoptin-treated, pEGFP-apoptin-treated or control-treated cells cultured in 6-well plates. Following transfection, cells were harvested and resuspended in lysis buffer, washed three times with ice-cold PBS and lysed in extraction buffer $(40 \mathrm{mmol} / \mathrm{l}$ Tris- $\mathrm{HCl}, \mathrm{pH} 7.5,150 \mathrm{mmol} / \mathrm{l}$ $\mathrm{KCl}, 1 \mathrm{mmol} / \mathrm{l}$ EDTA, $1 \%$ Triton $\mathrm{X}-100,100 \mathrm{mmol} / 1 \mathrm{NaVO}_{3}$, $1 \mathrm{mmol} / 1 \mathrm{PMSF}$ ) supplemented with the protease inhibitor cocktail. Proteins $(60 \mu \mathrm{g})$ were separated by $10 \%$ SDS-PAGE and transferred to PVDF membranes. The membranes were blocked with $10 \%$ non-fat milk in Tris-buffered saline (TBS) at $37^{\circ} \mathrm{C}$ for $3 \mathrm{~h}$ and then incubated with mouse anti-Flag antibody $(1: 1,000)$, mouse anti-GFP antibody $(1: 1,000)$ or mouse anti- $\beta$-actin antibody $(1: 2,000)$ in TBS containing $10 \%$ non-fat milk for $12 \mathrm{~h}$ at $4^{\circ} \mathrm{C}$. Horseradish peroxidaselinked anti-mouse $\operatorname{IgG}(1: 5,000)$ was used as a secondary antibody (in TBS containing 10\% non-fat milk for $3 \mathrm{~h}$ at room temperature), and antigen-antibody complexes were detected using an enhanced chemiluminescence kit (ECL Plus, Amersham, Freiburg, Germany). Densitometry values for western blotting and antibody array experiments were estimated by the ImageQuant TL software (GE Healthcare, Buckinghamshire, UK) and expressed as arbitrary units (a.u.). Multiple film exposures were used to verify the linearity 
A

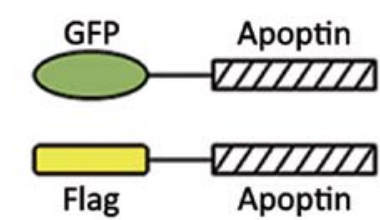

C

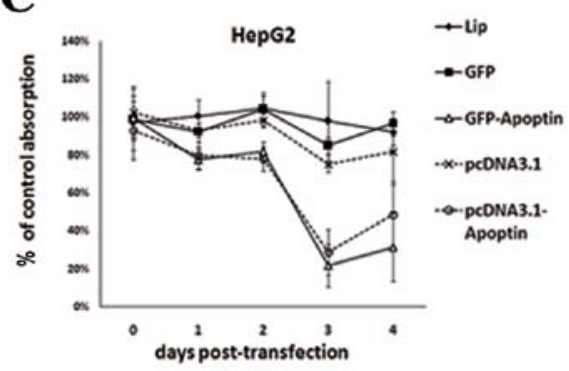

D
B
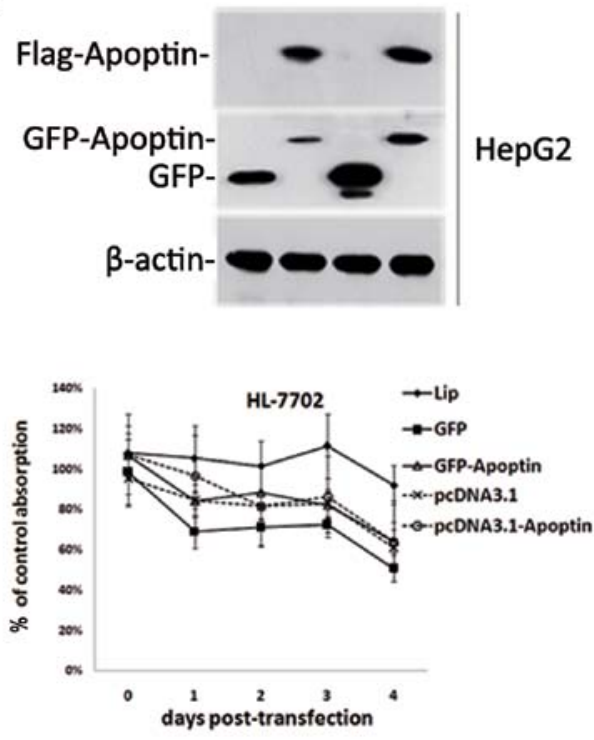

DAPI

Merge

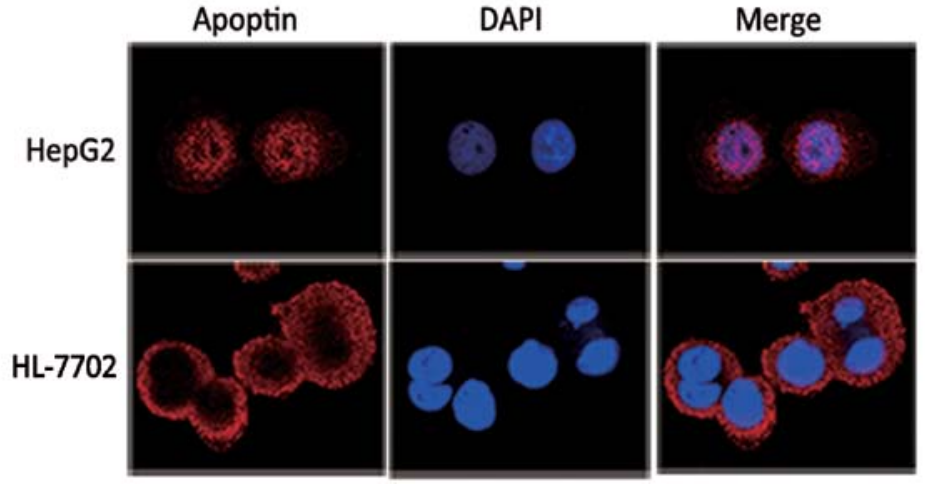

Figure 1. Apoptin-induced specific killing of HCC cells. (A) Schematic representations of the N-terminal Flag- or GFP-tagged apoptin expression plasmids. (B) HepG2 cells were transfected with Flag- or GFP-tagged apoptin expression plasmids and $48 \mathrm{~h}$ later, the expression of apoptin was detected by western blot analysis with an anti-Flag or anti-GFP antibody. (C) Effects of apoptin were determined by an MTT assay. HepG2 and HL-7702 cells were transfected with pcDNA3.1-Flag-apoptin/pEGFP-apoptin or pcDNA3.1/pEGFP. The cell viability was quantified with the MTT assay. Points, mean; bars, SE. "P<0.05, significant difference vs. control. The Y-axis, inhibiting rate, was determined in the assay. The assay was repeated at least three times in duplicate. (D) HepG2 and HL-7702 cells were transfected with pcDNA-Flag-apoptin. After $24 \mathrm{~h}$, cells were fixed, immunostained with the anti-Flag antibody and the Cy3-conjugated secondary antibody, and then visualized by a fluorescence microscope. Cells were also stained with DAPI to reveal the nuclei.

of the samples analyzed and to avoid saturation of the film. All antibodies used in the study were purchased from Cell Signaling Technology.

Immunoprecipitation. Cells were seeded in 10-cm dishes and transfected with pcDNA3.1-Flag-apoptin/pEGFP-apoptin vector or pcDNA3.1/pEGFP empty vector. At $24 \mathrm{~h}$ after transfection, cells were washed three times with ice-cold PBS, lysed in $1 \mathrm{ml}$ of ice-cold cell lysis buffer $(40 \mathrm{mmol} / 1$ HEPES, $120 \mathrm{mmol} / 1 \mathrm{NaCl}, 1 \%$ Triton X-100, $10 \mathrm{mmol} / \mathrm{l}$ pyrophosphate, $10 \mathrm{mmol} / 1$ glycerophosphate, $50 \mathrm{mmol} / 1 \mathrm{NaF}$, $1.5 \mathrm{mmol} / 1 \mathrm{Na}_{3} \mathrm{VO}_{4}, 1 \mathrm{mmol} / 1$ EDTA, and complete protease inhibitor cocktail, $\mathrm{pH} \mathrm{7.5)}$ on ice for $30 \mathrm{~min}$. The cell debris was removed, and anti-GFP (CST, UK) or anti-CDK1 antibody (CST, UK) was added to the cell lysate for $30 \mathrm{~min}$ at room temperature under constant agitation. A total of $20 \mu \mathrm{l}$ of washed Bio-Adembeads PAG (Invitrogen) was added to the cell lysate with an antibody for $2-3 \mathrm{~h}$ at $4^{\circ} \mathrm{C}$ and complexes were collected using a magnet rack. Then, the immunocomplex was analyzed by a western blot assay.
Immunofluorescence. Cells were plated on coverslips in a 12 -well plate the day before transfection. Transfection was performed using Lipofectamine 2000 (Invitrogen). At $24 \mathrm{~h}$ after transfection, cells were fixed with $4 \%$ paraformaldehyde, permeabilized in $0.1 \%$ Triton $\mathrm{X}-100$, blocked in $3 \%$ bovine serum albumin (BSA), and incubated with mouse anti-Flag monoclonal antibody or anti-CDK1 antibody (CST, USA) for $1 \mathrm{~h}$ and Cy3-red or FITC-green IgG secondary antibody (KPL, Gaithersburg, MD, USA) for $1 \mathrm{~h}$. The cells were washed and covered with DAPI (4,6-diamidino-2-phenylindole) mounting medium (Vector Laboratories, Cambridgeshire, UK) and visualized by using a fluorescence microscope (Leica spII).

\section{Results}

Apoptin-induced specific killing of HepG2 cells. HepG2 and HL-7702 cells were transfected with pcDNA3.1-Flagapoptin/pEGFP-apoptin or pcDNA3.1/pEGFP to investigate whether apoptin had differential cytotoxicity toward HCC and normal liver cells. First, western blot analysis was used 


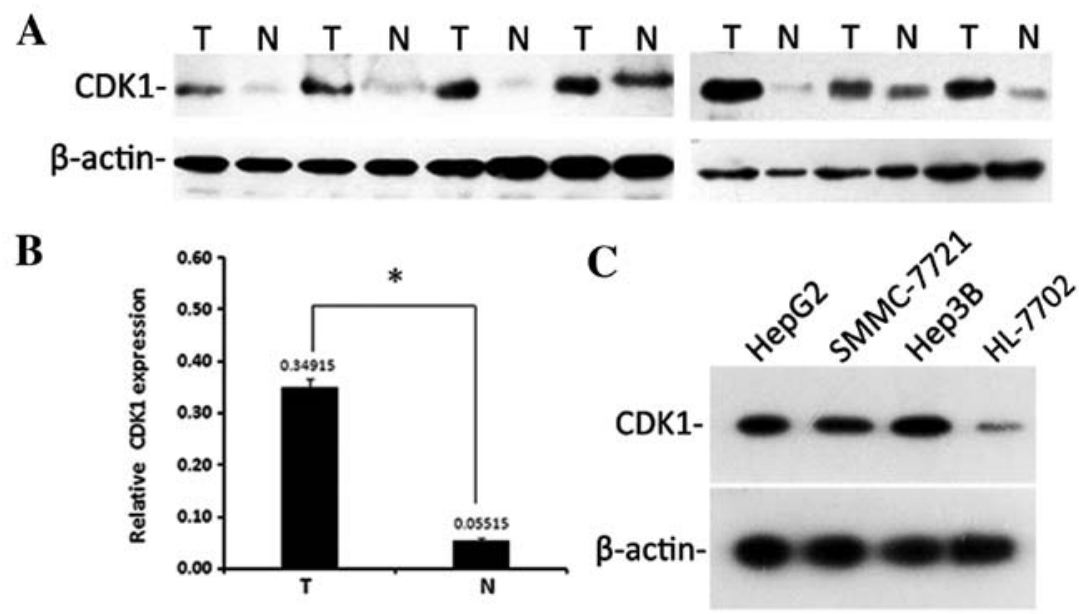

Figure 2. CDK1 expression in human hepatic cancer tissues and cell lines. (A) The expression levels of CDK1 were determined in 48 pairs of human hepatic cancer tissues (T) and their corresponding adjacent non-tumor tissues (N) by western blot analysis with an anti-CDK1 antibody. (B) Real-time PCR analyses were performed on 48 pairs of human hepatic cancer tissues and their corresponding adjacent non-tumor tissues to determine the expression levels of CDK1. (C) The expression levels of CDK1 were detected in HCC cell lines HepG2, Hep3B, SMMC-7721 and the normal liver cell line HL-7702 by western blot analysis with an anti-CDK1 antibody.

A

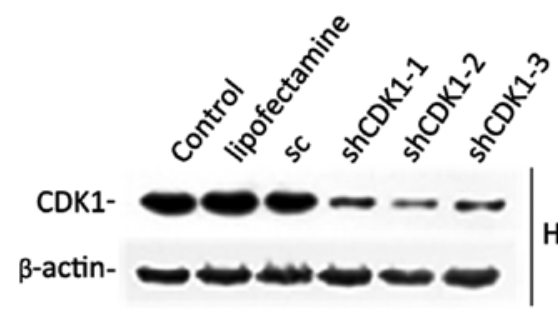

C

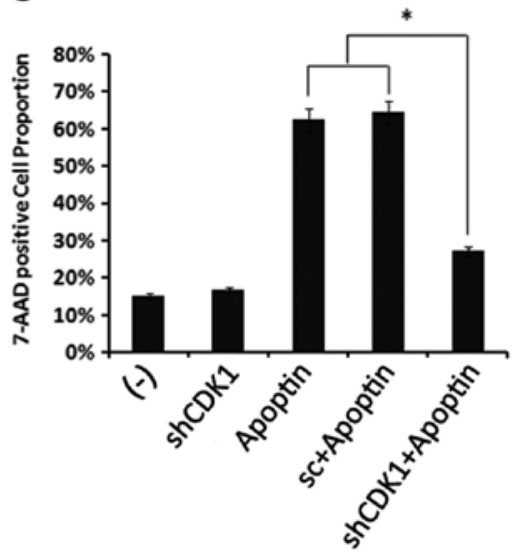

B
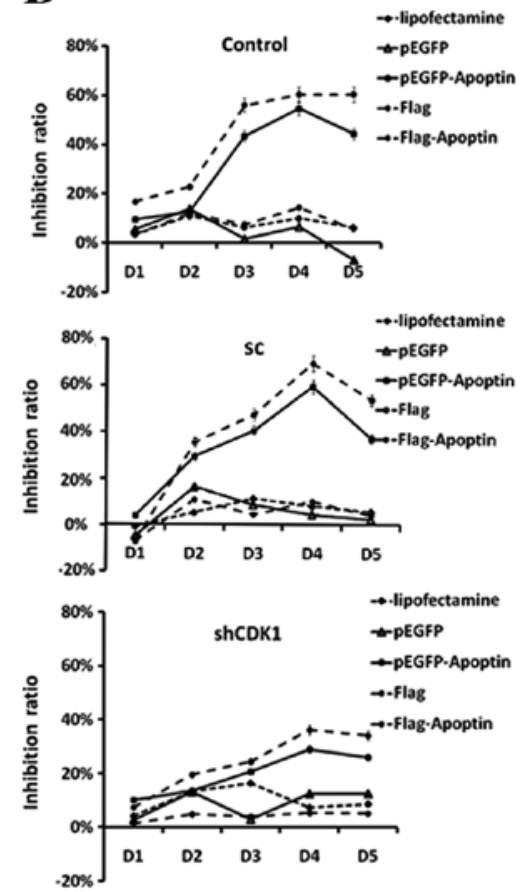

Figure 3. shCDK1-mediated knockdown of CDK1 activity effectively inhibits apoptin-induced apoptosis. (A) Western blotting demonstrated successful knockdown of endogenous CDK1 protein by shRNA. HepG2 cells were transfected with shCDK1. After $48 \mathrm{~h}$, the expression of CDK1 was detected by western blotting with an anti-CDK1 antibody. (B) HepG2 cells were co-transfected with pcDNA3.1-Flag-apoptin/pEGFP-apoptin or pcDNA3.1/pEGFP and a lentiviral vector expressing CDK1 shRNA. The cell viability was quantified with the MTT assay. Points, mean; bars, SE. * $\mathrm{P}<0.05$, significant difference vs. control. The Y-axis, inhibiting rate, was determined in the assay. The assay was repeated at least three times in duplicate. (C) Flow cytometry was performed after transfection with pcDNA3.1-Flag-apoptin, pEGFP- apoptin and a lentiviral vector expressing CDK1 shRNA. The induction rate of apoptosis was calculated as percentage of PE+/7-AAD+ cells in HepG2. Early apoptotic cells, late apoptotic cells and necrotic cells were measured according to the manufacturer's protocol. ${ }^{*} \mathrm{P}<0.05$ significant difference vs. control.

to detect the expression of apoptin (Fig. 1B). Using the MTT assay, the efficacy of tumor-specific killing activity was evaluated on days 1, 2, 3, 4 and 5 after transfection. As shown in Fig. 1C, the cell viability of HepG2 cells was significantly decreased after transfection with pcDNA3.1-Flag-apoptin or pEGFP-apoptin as compared to the control, whereas the normal liver cell line HL-7702 was not affected by apoptin. Expression of apoptin-induced apoptosis in HepG2 cells had 

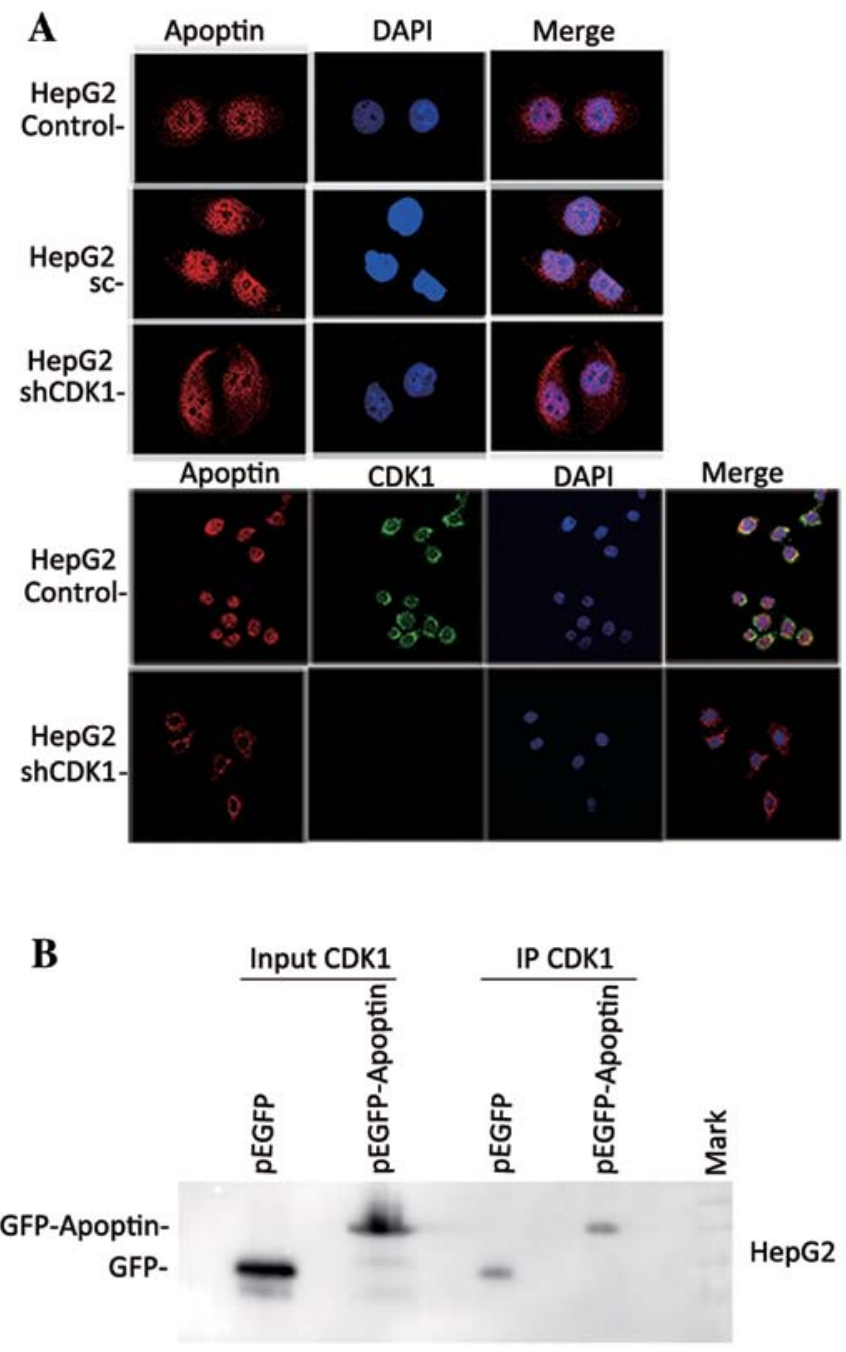

Figure 4. shCDK1-mediated knockdown of CDK1 affects the subcellular localization of apoptin. (A) HepG2 cells were co-transfected with pcDNA3.1Flag-apoptin and a lentiviral vector expressing CDK1 shRNA. After $24 \mathrm{~h}$, the cells were fixed, immunostained with the anti-Flag or anti-CDK1 antibody and a Cy3- or FITC-conjugated secondary antibody, and then visualized by a fluorescence microscope. Cells were also stained with DAPI to reveal the nuclei. (B) HepG2 cells were transfected with a pEGFP-apoptin expression construct. After $48 \mathrm{~h}$, apoptin was immunoprecipitated with an anti-GFP antibody, and the expression of CDK1 or apoptin was analyzed by western blotting.

little or no cytotoxic effect on HL-7702 cells, which indicates that apoptin can be applied as a potential therapeutic target in HCC treatment. To further examine the subcellular localization of apoptin in HepG2 and HL-7702 cells, the cells were transfected with pcDNA3.1-Flag-apoptin, and the intracellular distribution of the expressed Flag-apoptin was observed by immunofluorescent microscopy 24 h later. Flag-apoptin exhibited the same intracellular localization as GFP-apoptin and native apoptin in HepG2 or HL-7702 cells; i.e., apoptin rapidly translocated to the nucleus in HepG2 cells, whereas it remained dispersed predominantly in the cytoplasm in HL-7702 cells (Fig. 1D).

CDK1 expression in human hepatic cancer tissues and cell lines. Western blot analysis and real-time PCR analyses were performed on 48 pairs of human hepatic cancer tissues and their corresponding adjacent non-tumor tissues to determine the expression levels of CDK1. All 48 pairs of cancer tissues showed a significantly higher CDK1 expression level than their matched non-tumor tissues $(\mathrm{P}<0.001$; Fig. 2A and $\mathrm{B})$. In addition, the extent of their activation varied significantly, suggesting that the pathways leading to the activation of CDK1 can be regulated independently. Similar results were also observed in the HCC cell lines HepG2, Hep3B, SMMC7721 and the normal liver cell line HL-7702 (Fig. 2C). These results indicated that the abnormal activation of CDK1 was associated with the cancerous or precancerous state. Therefore, the dysregulation of this cellular pathway may be involved in the activation process of apoptin-induced tumorspecific apoptosis.

Knockdown of CDK1 effectively inhibits apoptin-induced apoptosis. To determine whether a causal link exists between CDK1 and apoptin-induced tumor-specific apoptosis, HepG2 cells were transfected with both pcDNA3.1-Flagapoptin/pEGFP-apoptin or pcDNA3.1/pEGFP and a lentiviral vector expressing CDK1 shRNA. Transfection with a construct expressing a scrambled shRNA was performed as a negative control. Western blotting demonstrated successful knockdown of endogenous CDK1 protein by shRNA (Fig. 3A). An MTT cell survival assay showed a strong reduction in the pro-apoptotic activity of apoptin after transfection with shRNA (Fig. 3B). Similar results were also observed by FACS analysis (Fig. 3C). These results collectively indicated that CDK1 was an important apoptin-interacting protein and played an essential role in the regulation of apoptin-induced apoptosis. However, other intricate pathways may also be involved, and further research is required to elucidate these pathways.

shRNA-mediated knockdown of CDK1 activity affects the subcellular localization of apoptin. The tumor-specific activity of apoptin is dependent on its ability to localize in the nucleus of tumor cells but not on its ability to localize in the nucleus of primary normal cells $(10,18)$. To confirm the association between CDK1 activity and the subcellular localization of apoptin, HepG2 cells were co-transfected with pcDNA3.1-Flag-apoptin and a lentiviral vector expressing CDK1 shRNA. At $24 \mathrm{~h}$ post-transfection, the nuclear translocation of apoptin was evaluated by immunofluorescent microscopy. Flag-apoptin exhibited the same intracellular distribution pattern in untransfected or scrambled shRNAtransfected parental cells as the native apoptin (Fig. 4A). However, in cells transfected with shRNA, Flag-apoptin relocalized predominantly to the cytoplasm (Fig. 4A). The above data confirmed that the activation of CDK1 may regulate the subcellular localization of apoptin and that the suppression of CDK1 activation had a significant effect on apoptin nuclear aggregation. To determine whether apoptin interacts with CDK1 in HepG2 cells, immunoprecipitation (IP) was performed. As shown in Fig. 4B, western blot analysis of the IP complexes confirmed co-immunoprecipitation of CDK1 with the GFP-apoptin fusion protein but not with GFP alone (Fig. 4B). These results suggest that there was definitive interaction between CDK1 and apoptin in HepG2 cells. 


\section{Discussion}

Several viral proteins that kill tumor cells have been identified. Apoptin is one of the family members of proline-rich proteins derived from the chicken anemia virus. Apoptin has an efficient apoptotic effect on cancer cells (9). An additional characteristic demonstrated by this chicken anemia virusderived protein is its tumor-specific cytotoxicity that differs from other chemo- or bio-therapeutic strategies $(6,7,9)$. These findings indicated that apoptin and its cellular interacting and regulatory targets may be important candidates for anticancer therapeutics. Apoptin-induced, tumor-specific apoptosis presumably requires additional interacting partners that may activate specific signaling pathways in cancer cells. Although a number of apoptin-interacting molecules have been identified, the molecular mechanism underlying the action of apoptin remains poorly understood. These interactions may be important for the nuclear localization of apoptin or its tumor-selective cytotoxicity (12-16). Based on our hypothesis, due to the tumor-specific cytotoxicity of apoptin, the mediator that interacts with apoptin in cancer cells should demonstrate a significantly different activity or expression in cancer cells as compared to normal cells. In this study, as well as in previous investigations, HCC tissues showed a significantly higher CDK1 expression than their matched non-tumor tissues. Similar results were also observed in HCC cell lines. These results suggested that CDK1 acts as a tumor-specific mediator, affecting apoptininduced cytotoxicity in HCC cells. CDK1 plays an important role in cell division (19), and several CDK1 substrates, such as histone $\mathrm{H} 1$ and $\mathrm{PI} 3 \mathrm{~K} / \mathrm{AKT}$, play crucial roles in cell cycle modulation $(20,21)$. CDKs form a family of Ser/Thr kinases that phosphorylate hundreds of protein substrates. $[\mathrm{S} / \mathrm{T} * \mathrm{PX}[\mathrm{K} / \mathrm{R}]$ is the classic recognition site of CDK1 (22-24) and, coincidentally, the amino acid sequences surrounding the phosphorylation site of apoptin is TTTPSR PR (25), which indicates that CDKs are candidate kinases for apoptin. Consistent with this finding, the results of our previous calculation using the KinasePhos software (http://kinasephos.mbc. nctu.edu.tw) suggested that CDKs, including CDK1, CDK2 and CDK4/6, are noteworthy candidates for interaction with apoptin. Several studies have shown an interaction between CDK2 and apoptin. Maddika et al (26) found that CDK2 is an important kinase in apoptin-induced apoptosis. In this study, to clarify the mechanism of the tumor-specific killing effect induced by apoptin, we investigated the interaction of apoptin with CDK1. Although our co-IP result confirmed that CDK1 interacted with apoptin, the detailed mechanism of the CDK1-apoptin interaction remains unclear, and further experiments are required to reveal this. CDK1 participates in a subset of apoptotic programs aside from cell cycle regulation $(17,27)$. However, there was no evidence to indicate the relevance between CDK1 and exogenous cell-killing proteins. Our study is the first to establish the relationship between CDK1 and apoptin and highlights a new mechanism of cell death induced by CDK1.

Apoptin is predominantly localized in the nucleus of cancer cells, whereas in normal cells, its nuclear accumulation is severely impaired $(10,11,18)$. Here, we provided evidence that the nuclear accumulation of apoptin in tumor cells is mediated by CDK1. We also investigated whether there is a causal link between CDK1 and apoptin-induced, tumor-specific apoptosis. A strong reduction in apoptininduced apoptosis was observed after successful knockdown of endogenous CDK1 protein by shRNA. These results suggested that CDK1 was an important protein interacting with apoptin and played a significant role in the regulation of apoptin-induced apoptosis. Previous studies have shown that the selective toxicity of apoptin is mainly attributed to its differential subcellular localization in tumor and normal cells (10). In cancer cells, apoptin is predominantly localized in the nucleus, whereas in normal cells, apoptin is detected in the cytoplasm $(10,11,18)$. Hence, the subcellular localization of apoptin could indirectly reflect its apoptotic activity. shRNA knockdown of CDK1 significantly reduced nuclear accumulation of apoptin in HepG2 cells, which further confirmed the association between the CDK1 activation and the cytotoxic activity of apoptin in HepG2 cells. Moreover, Lee et al (28) asserted that GFP-apoptin did not exhibit the same intracellular distribution pattern as wild-type apoptin. By contrast, our results showed the same intracellular localization of Flagapoptin and GFP-apoptin as wild-type apoptin in HCC and normal liver cells. Our results are consistent with previous findings (29). A different cell line would be a possible factor causing this divergence. However, the present study identified a previously unrecognized mechanism of interaction between CDK1 and apoptin.

In conclusion, the abnormal activation of CDK1 was generally associated with the cancerous or precancerous state. A definitive interaction between CDK1 and apoptin was also detected; CDK1 may regulate the subcellular localization of apoptin. Our study provides a novel mechanism for apoptin regulation involving the CDK1 pathway.

\section{Acknowledgements}

This study was supported by the China National Natural Scientific Foundation (no. 81071692 and 81272488), the Shaanxi Province Natural Scientific Foundation (no. 2010JM4021) and the First Affiliated Hospital of Xi'an Jiaotong University Medical College Foundation (no. 2009-13).

\section{References}

1. Zhang Y, Wang S, Li D, et al: A systems biology-based classifier for hepatocellular carcinoma diagnosis. PloS One 6: e22426, 2011.

2. Almogy G, Lieberman S, Gips M, et al: Clinical outcomes of surgical resections for primary liver sarcoma in adults: results from a single centre. Eur J Surg Oncol 30: 421-427, 2004.

3. Tanaka S and Arii S: Molecularly targeted therapy for hepatocellular carcinoma. Cancer Sci 100: 1-8, 2009.

4. Noteborn MH, Todd D, Verschueren CA, et al: A single chicken anemia virus protein induces apoptosis. J Virol 68: 346-351, 1994.

5. Noteborn MH, de Boer GF, van Roozelaar DJ, et al: Characterization of cloned chicken anemia virus DNA that contains all elements for the infectious replication cycle. J Virol 65: 3131-3139, 1991.

6. Tavassoli M, Guelen L, Luxon BA and Gaken J: Apoptin: specific killer of tumor cells? Apoptosis 10: 717-724, 2005.

7. Noteborn MH: Proteins selectively killing tumor cells. Eur J Pharmacol 625: 165-173, 2009.

8. Guelen L, Paterson H, Gaken J, Meyers M, Farzaneh F and Tavassoli M: TAT-apoptin is efficiently delivered and induces apoptosis in cancer cells. Oncogene 23: 1153-1165, 2004. 
9. Danen-Van Oorschot AA, Fischer DF, Grimbergen JM, et al Apoptin induces apoptosis in human transformed and malignant cells but not in normal cells. Proc Natl Acad Sci USA 94 5843-5847, 1997.

10. Danen-Van Oorschot AA, Zhang YH, Leliveld SR, et al: Importance of nuclear localization of apoptin for tumor-specific induction of apoptosis. J Biol Chem 278: 27729-27736, 2003

11. Heilman DW, Teodoro JG and Green MR: Apoptin nucleocytoplasmic shuttling is required for cell type-specific localization, apoptosis, and recruitment of the anaphase-promoting complex/ cyclosome to PML bodies. J Virol 80: 7535-7545, 2006.

12. Maddika S, Wiechec E, Ande SR, et al: Interaction with PI3-kinase contributes to the cytotoxic activity of apoptin. Oncogene 27: 3060-3065, 2008.

13. Maddika S, Bay GH, Kroczak TJ, et al: Akt is transferred to the nucleus of cells treated with apoptin, and it participates in apoptin-induced cell death. Cell Prolif 40: 835-848, 2007.

14. Jiang J, Cole D, Westwood N, et al: Crucial roles for protein kinase $\mathrm{C}$ isoforms in tumor-specific killing by apoptin. Cancer Res 70: 7242-7252, 2010.

15. Teodoro JG, Heilman DW, Parker AE and Green MR: The viral protein Apoptin associates with the anaphase-promoting complex to induce G2/M arrest and apoptosis in the absence of p53. Genes Dev 18: 1952-1957, 2004.

16. Janssen K, Hofmann TG, Jans DA, Hay RT, Schulze-Osthoff K and Fischer U: Apoptin is modified by SUMO conjugation and targeted to promyelocytic leukemia protein nuclear bodies. Oncogene 26: 1557-1566, 2007.

17. Shi L, Nishioka WK, Th'ng J, Bradbury EM, Litchfield DW and Greenberg AH: Premature p34cdc2 activation required for apoptosis. Science 263: 1143-1145, 1994.

18. Kuusisto HV, Wagstaff KM, Alvisi G and Jans DA: The C-terminus of apoptin represents a unique tumor cell-enhanced nuclear targeting module. Int J Cancer 123: 2965-2969, 2008.
19. Murray AW: Recycling the cell cycle: cyclins revisited. Cell 116: 221-234, 2004

20. Brown NR, Noble ME, Endicott JA and Johnson LN: The structural basis for specificity of substrate and recruitment peptides for cyclin-dependent kinases. Nat Cell Biol 1: 438-443, 1999.

21. Loog M and Morgan DO: Cyclin specificity in the phosphorylation of cyclin-dependent kinase substrates. Nature 434: 104-108, 2005.

22. Brotherton DH, Dhanaraj V, Wick S, et al: Crystal structure of the complex of the cyclin D-dependent kinase Cdk6 bound to the cell-cycle inhibitor p19INK4d. Nature 395: 244-250, 1998.

23. Jeffrey PD, Russo AA, Polyak K, et al: Mechanism of CDK activation revealed by the structure of a cyclinA-CDK 2 complex. Nature 376: 313-320, 1995.

24. Honda R, Lowe ED, Dubinina E, et al: The structure of cyclin E1/ CDK2: implications for CDK2 activation and CDK2-independent roles. EMBO J 24: 452-463, 2005.

25. Los M, Panigrahi S, Rashedi I, et al: Apoptin, a tumor-selective killer. Biochim Biophys Acta 1793: 1335-1342, 2009.

26. Maddika S, Panigrahi S, Wiechec E, et al: Unscheduled Akt-triggered activation of cyclin-dependent kinase 2 as a key effector mechanism of apoptin's anticancer toxicity. Mol Cell Biol 29: 1235-1248, 2009.

27. Golsteyn RM: Cdk1 and Cdk2 complexes (cyclin dependent kinases) in apoptosis: a role beyond the cell cycle. Cancer Lett 217: 129-138, 2005.

28. Lee YH, Cheng CM, Chang YF, Wang TY and Yuo CY: Apoptin T108 phosphorylation is not required for its tumor-specific nuclear localization but partially affects its apoptotic activity. Biochem Biophys Res Commun 354: 391-395, 2007.

29. Poon IK, Oro C, Dias MM, Zhang J and Jans DA: Apoptin nuclear accumulation is modulated by a CRM1-recognized nuclear export signal that is active in normal but not in tumor cells. Cancer Res 65: 7059-7064, 2005. 